\title{
Recycling universe
}

\author{
Jaume Garriga \\ IFAE, Departament de Fisica, Universitat Autonoma de Barcelona, 08193 Bellaterra (Barcelona), Spain
}

\author{
Alexander Vilenkin \\ Institute of Cosmology, Department of Physics and Astronomy, Tufts University, Medford, Massachusetts 02155
}

(Received 29 July 1997; published 12 January 1998)

\begin{abstract}
If the effective cosmological constant is nonzero, our observable universe may enter a stage of exponential expansion. In such a case, regions of it may tunnel back to the false vacuum of an inflaton scalar field, and inflation with a high expansion rate may resume in those regions. An "ideal" eternal observer would then witness an infinite succession of cycles from false vacuum to true, and back. Within each cycle, the entire history of a hot universe would be replayed. If there were several minima of the inflaton potential, our ideal observer would visit each one of these minima with a frequency which depends on the shape of the potential. We generalize the formalism of stochastic inflation to analyze the global structure of the universe when this "recycling", process is taken into account. [S0556-2821(98)02904-X]
\end{abstract}

PACS number(s): 98.80.Hw, 98.80.Bp, 98.80.Cq

\section{INTRODUCTION}

Inflationary models are designed to produce a universe which is sufficiently homogeneous on all observable scales [1]. However, on much larger scales the universe is expected to be extremely inhomogeneous. The evolution of the field $\phi$, whose vacuum energy drives inflation, is influenced by quantum fluctuations. These fluctuations can be pictured as a random walk of $\phi$ superimposed on its slow roll down the slope of its potential. As a result, thermalization of the vacuum energy does not occur simultaneously everywhere in the universe, and at any time there are parts of the universe that are still inflating [2,3].

On very large scales, the universe is expected to consist of isolated thermalized regions embedded in the inflating background. The boundaries of the thermalized regions expand into this background, and new regions are constantly being formed, but the high expansion rate of the intervening inflating domains prevents these regions from filling up the universe. Thermalization inevitably occurs at any given comoving location, and the comoving volume of the inflating regions decreases exponentially with time. At the same time, the physical volume of these regions is exponentially growing. The geometry of the inflating regions is that of a selfsimilar fractal of dimension $d<3$ [4]. It is illustrated in Fig. 1 for the case of "open" inflation, where the false vacuum decay occurs through bubble nucleation [5,6]. For "new" or "chaotic" inflation the picture would be similar, except the thermalized regions would have irregular shapes.

In the present paper we are going to argue that this picture of the superlarge-scale structure of the universe can be significantly modified by quantum fluctuations that bring localized parts of already thermalized regions, such as our observable universe, back to the inflating false-vacuum state. The modification is particularly important in models where the post-thermalization true vacuum is characterized by a positive vacuum energy (cosmological constant). In this case the thermalized regions asymptotically approach de Sitter geometry, and the rate of fluctuations back to the false vacuum (per unit spacetime volume) approaches a constant. Even with an exceedingly small rate, the probability for true vacuum to survive at any comoving location is exponentially decreasing with time. Hence, (almost) all the comoving volume of thermalized regions will eventually be recycled back to the inflationary phase. Each nucleated false vacuum region will serve as a seed for a new eternally inflating domain, whose internal structure will resemble that shown in Fig. 1. The thermalized regions formed in this domain will in turn produce new false vacuum seeds, etc. We call this kind of model a recycling universe.

Quantum nucleation of regions with a higher energy density cannot occur from a flat-spacetime vacuum characterized by a vanishing cosmological constant: such processes are forbidden by energy conservation. However, upward fluctuations of this kind can occur in an expanding cosmological background, and have been previously discussed by a number of authors [7,2,3,8-11]. The most relevant for our purposes here is the paper by Lee and Weinberg [11] who considered a model of a scalar field $\phi$ with a potential $V(\phi)$

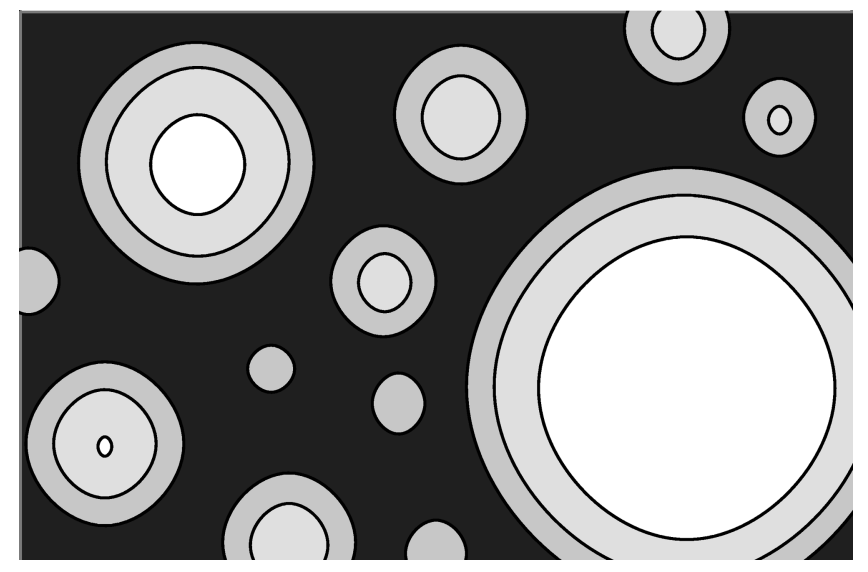

FIG. 1. True vacuum bubbles (white) nucleating in false vacuum (black). The shaded rings represent slow roll regions (external ring) and matter or radiation dominated regions (internal ring). 


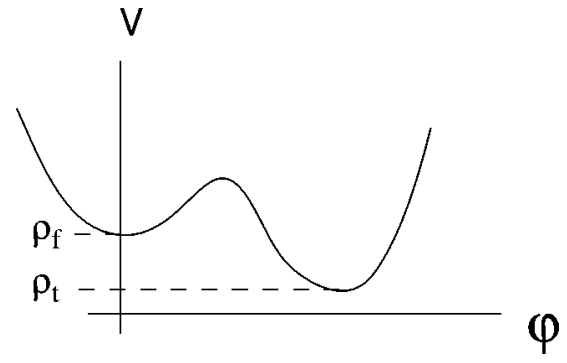

FIG. 2. Self interaction potential for the "tunneling" scalar field. The energy densities in false and true vacua, denoted as $\rho_{f}$ and $\rho_{t}$, act as an effective cosmological constant.

shown in Fig. 2. Note that both false and true vacua have positive energy densities, $\rho_{f}>\rho_{t}>0$. It has been known for some time that the high-energy false vacuum at $\phi=0$ can decay by nucleation of true vacuum bubbles. The corresponding instanton ("bounce') has been found by Coleman and De Luccia [12]. The bubble nucleation rate is given by

$$
\Gamma_{f \rightarrow t}=A \exp \left[-S_{b}+S_{f}\right]
$$

where $S_{b}$ is the bounce action and $S_{f}=-3 / 8 \rho_{f}$ is the action of the Euclideanized false-vacuum de Sitter space (we use Planck units throughout the paper). Lee and Weinberg conjectured that the same instanton also describes the inverse process of true vacuum decay, where false vacuum bubbles nucleate in a true vacuum background. The nucleation rate suggested by Eq. (1) is

$$
\Gamma_{t \rightarrow f}=A \exp \left[-S_{b}+S_{t}\right]
$$

with $S_{t}=-3 / 8 \rho_{t}$. Lee and Weinberg argued that the preexponential factors in Eqs. (1) and (2) are the same. These conjectures were later verified [13] in the case of $(1+1)$-dimensional universes, where bubble nucleation can be identified with the production of particle-antiparticle pairs. Note that the rate (2) vanishes if the true vacuum has zero energy.

As it stands, the potential in Fig. 2 is not suitable for inflationary cosmology. This potential has no slow-roll region, so most of the vacuum energy remains in domain walls and never gets thermalized. We shall see, however, that models incorporating both realistic inflation and true vacuum decay can be constructed by a trivial modification of "open" inflationary models. Moreover, we shall argue that nucleation of inflating regions is possible even with the simplest slow-roll potentials, for which the Coleman-de Luccia instanton does not exist.

The recycling nature of inflationary universe may have important implications for the question of whether or not the universe had a beginning in time. As we already mentioned, inflation is generically eternal to the future, so it is natural to ask if the inflationary models can be continued into the infinite past, resulting in a "steady-state" nonsingular cosmology. This possibility was discussed in the early 1980s, soon after the inflationary scenario was proposed, with the conclusion that the idea could not be implemented in the simplest model in which the inflating universe is described by an exact de Sitter space [14,2]. A more general proof of impossibility of steady state inflation was given in Refs. [15-17], but we shall see later in this paper that some of the assumptions made in the proof do not apply in the case of a recycling universe. The question of the necessity of the beginning is therefore reopened.

Recycling may also be relevant to the question of making predictions in an inflationary universe. Recently, there have been a number of attempts [18-23] to find probability distributions for cosmological parameters such as the effective cosmological constant $\Lambda$ or the density parameter $\Omega$. These "predictions" are based on the principle of mediocrity [20,24-26], by which we are most likely to live in the most abundant type of civilization that can result from the thermalization of a false vacuum. However, in the inflationary universe, there will be an infinite number of infinite thermalized regions, and one faces the difficulty of comparing infinities $[19,18,27]$. Regularization procedures were introduced in $[21,28]$ to deal with this problem, but these cannot be directly applied to a recycling universe.

The paper is organized as follows. In the next section we shall give some examples of inflationary models which allow nucleation of false vacuum bubbles. The geometry of the nucleated bubbles will be analyzed in Sec. III. In Sec. IV we shall discuss the implications of the recycling universe model for the question of the beginning of the universe. The superlarge-scale structure of a recycling universe will be studied in Sec. V using the methods of stochastic inflation. The issue of predictions will be discussed in Sec. VI, and our conclusions will be summarized in Sec. VII.

\section{MODELS}

In all realistic inflationary models, the potential of the inflaton field $\phi$ is required to have a sufficiently flat slow-roll region in which

$$
\left|V^{\prime \prime}(\phi)\right| \ll H^{2}
$$

Here, $H$ is the expansion rate and $H^{-1}$ is the horizon size corresponding to the vacuum energy $V(\phi)$,

$$
H^{2}=8 \pi V(\phi) / 3
$$

On the other hand, Coleman-de Luccia-type solutions for vacuum bubbles exist only when the potential is sufficiently curved near the barrier separating true and false vacua [29],

$$
\left|V^{\prime \prime}(\phi)\right| \gtrsim H^{2}
$$

The meaning of this condition is easy to understand. The bubble wall thickness is $\delta \sim\left|V^{\prime \prime}\right|^{-1 / 2}$, and if (5) is not satisfied, then the wall is much thicker than the horizon. Such walls cannot exist as coherent structures and are spread by the expansion of the universe. False vacuum bubbles of Coleman-de Luccia type are, therefore, impossible if the slow roll condition (3) is valid everywhere in the inflationary range of $\phi$.

A similar problem arises in the "open', inflation scenario, where false vacuum decay through bubble nucleation is followed by a period of slow roll in bubble interiors $[5,6]$. One way to deal with this problem is to consider a two-field model, with one field doing the tunneling and the other doing the slow roll [6]. The potential can be chosen as 


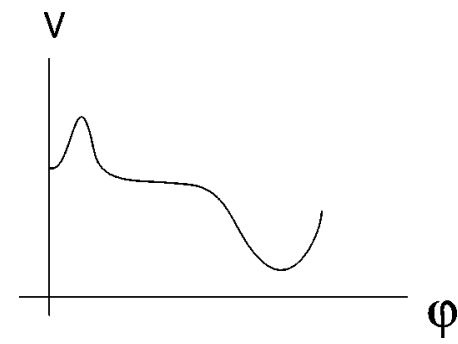

FIG. 3. The potential for the scalar field in the one field model of open inflation. The sharp barrier followed by a flat plateau gives this potential a somewhat unnatural appearance.

$$
U(\chi, \phi)=V_{1}(\chi)+\chi^{2} V_{2}(\phi)
$$

Here, $\chi$ is the tunneling field and the potential $V_{1}(\chi)$ has the form as in Fig. 2 with a metastable minimum at $\chi=0$ and a true minimum at $\chi=\eta_{\chi}$. The full potential $U(\chi, \phi)$ is independent of $\phi$ (has a flat direction) at $\chi=0$, and as a result the expansion rate in the false vacuum is also independent of $\phi$. The potential $V_{2}(\phi)$ is assumed to have a slow-roll range and a minimum at $\phi=\eta_{\phi}$ with $V_{2}\left(\eta_{\phi}\right)=0$, at which thermalization eventually occurs. In a variant of this model [6], the two fields can be taken to represent the radial and angular parts of a single complex field, $\Phi=\chi e^{i \phi}$.

The stage for open inflation is set by inflating false vacuum with energy density $\rho_{f}=V_{1}(0)$. Nucleating bubbles expand into this background, but because of the high expansion rate of the intervening false vacuum regions, bubble collisions are rare. The interior geometry of each bubble is that of an open Robertson-Walker universe. The bubbles have different initial values of $\phi$, and if this value falls in the slow-roll range of the potential, then there is a period of inflation inside the corresponding bubble. Inflation is followed by thermalization and standard cosmological evolution, but since we assumed a nonzero cosmological constant, the bubble interiors are eventually dominated by the true vacuum energy, $\rho_{t}=V_{1}\left(\eta_{\chi}\right)$. False vacuum bubbles will now be formed in the true vacuum background, resulting in the endless succession of stages of the recycling universe.

It should be noted that tunneling back to the false vacuum can occur not only from the true vacuum $(\chi, \phi)=\left(\eta_{\chi}, \eta_{\phi}\right)$, but also from the slow-roll, as well as radiation and matterdominated periods. In fact, the rate of false vacuum bubble nucleation is expected to be the highest during the slow-roll inflation [due to the higher energy density at that time, see Eq. (2)]. However, since all these periods last only for a finite time, and the rate of false vacuum bubble formation is extremely low, only a tiny fraction of the comoving volume will be affected by such processes. On the other hand, the true-vacuum-dominated stage persists indefinitely, and practically all the comoving volume will be recycled by bubbles nucleating in the true vacuum.

An alternative to the two-field model (6) is a model of a single scalar field $\phi$ with a potential of the form shown in Fig. 3 [5]. The false vacuum at $\phi=0$ is separated from the slow-roll region by a sharp barrier. (The coexistence of flat and highly curved regions in the same potential is a somewhat unnatural feature of this model.) The field $\phi$ tunnels through the barrier and after a period of slow roll, ends up in the true vacuum, which we assume to have a nonzero energy

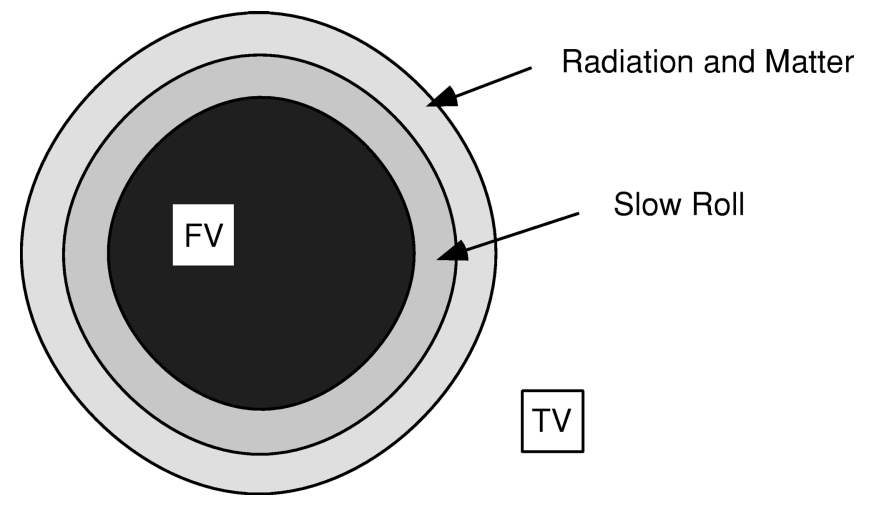

FIG. 4. A false vacuum bubble (black) nucleating in true vacuum (white). Regions of slow roll and of matter and radiation domination surrounding the bubble are indicated.

density, $\rho_{t}>0$. An important difference of this model from that of Eq. (6) is that now, in order to tunnel from true to false vacuum, the field $\phi$ has to go across the whole slowroll region. False vacuum bubbles will therefore consist of a false vacuum core surrounded by a domain wall, which is in turn surrounded by layers of slow roll, radiation, and matterdominated regions (see Fig. 4).

Intuitively, we would expect that the nucleation of such a complicated structure should be extremely unlikely, and thus the nucleation rate of false vacuum bubbles in this one-field model should be strongly suppressed compared to the twofield model (6). In the model corresponding to Fig. 3, the Coleman-de Luccia instanton crosses the barrier but not the plateau. This is required in open models to ensure a second period of slow roll inflation solving the flatness problem. In the spirit of Lee and Weinberg [11], we could naively reinterpret this instanton as describing tunneling from true vacuum to false. But since the instanton does not really interpolate between both minima, the interpretation seems somewhat unjustified. Incidentally, in curved space the instantons never exactly interpolate between both minima, but for a potential of the type represented in Fig. 3 the situation is clearly more extreme. Therefore, an estimate of the tunneling rate a la Lee-Weinberg seems questionable in this case. It should be remembered also that the use of Euclidean methods in de Sitter space has never been justified from first principles, and therefore the results obtained using these methods should be taken with caution [8-10]. This issue needs further investigation, but we will not attempt to address it in the present paper. The specific value of false vacuum bubble nucleation rate will not be important for our conclusions, as long as this rate is nonzero.

If the barrier in the inflaton potential is too wide to satisfy the condition (5), then the Coleman-de Luccia instanton does not exist. However, there is always a homogeneous Hawking-Moss instanton [7] in which $\phi$ takes the value $\phi_{b}$ corresponding to the top of the barrier. This instanton is usually interpreted as describing quantum tunneling from false vacuum to the top of the barrier in a horizon-size region. (Coleman-de Luccia instanton reduces to that of Hawking and Moss as the barrier width is increased.) Again, in the spirit of Lee and Weinberg [11], we can interpret the same instanton as describing tunneling from true vacuum to the top of the barrier. The corresponding nucleation rate is 


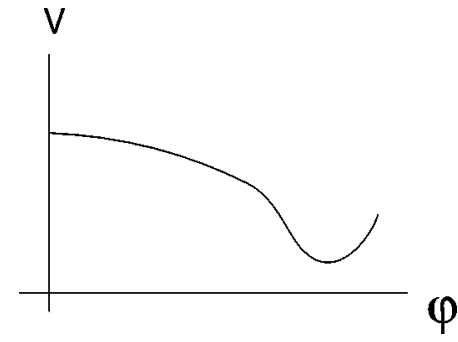

FIG. 5. The inflaton potential for the case of new inflation.

$$
\Gamma \propto \exp \left[-S_{H M}+S_{t}\right]
$$

where $S_{H M}=-3 / 8 \rho_{b}$ and $\rho_{b}=V\left(\phi_{b}\right)$.

In models of "new" inflation, the generic potential is illustrated in Fig. 5. There is no barrier in this case, but still there is a Hawking-Moss instanton with $\phi_{b}$ corresponding to the maximum of the potential. There are also approximate homogeneous instanton solutions with $\phi$ sufficiently close to the maximum. Such approximate instantons also exist in models of "chaotic" inflation where the potential may have no maxima. A constant field $\phi$ is a good approximation as long as the evolution of $\phi$ is slow on the Hubble scale $H^{-1}$, that is, in the slow roll range. The Euclidean expression for the tunneling rate from true vacuum to $\phi$ in this range is given by Eq. (7) with $S_{H M}=-3 / 8 V(\phi)$.

Even in flat space, there are instantons which describe tunneling without barriers [30]. Jensen and Steinhardt have argued that when gravity is included, these instantons are subdominant with respect to either the Hawking-Moss or the Coleman-de Luccia modes [31].

Once again, we find the formula (7) somewhat suspicious, and emphasize the need for a derivation of the nucleation rate without relying on Euclidean methods. Linde [10] has given an estimate of the probability of tunneling to false vacuum on rather general grounds. In de Sitter space, a field $\phi$ fluctuates on scales bigger or comparable to the Hubble radius around a local minimum $\phi_{t}$ of the potential with amplitude given by [32]

$$
\sigma^{2}=\left\langle\left(\phi-\phi_{t}\right)^{2}\right\rangle \approx \frac{3 H^{4}}{8 \pi^{2} m^{2}} .
$$

Strictly speaking, the result is only true for a free field and for small fluctuations, so that the mass $m$ and the expansion rate $H$ are well defined. Extrapolating to the case of an interacting field and ignoring the gravitational backreaction of the fluctuations on the expansion rate, the probability that a region of size $\sim H^{-1}$ will tunnel from $\phi_{t}$ to a different value $\phi$ sufficiently close to the maximum of $V(\phi)$ can be estimated as

$$
P \propto \exp \left[-\left(\phi-\phi_{t}\right)^{2} / 2 \sigma^{2}\right]
$$

For the case of a quadratic potential with $m^{2} \ll H^{2}$ and $V(\phi)-V\left(\phi_{t}\right) \ll V\left(\phi_{t}\right)$, the exponent in the previous expression reproduces the exponent $\left[-S_{H M}+S_{t}\right]$ which appears in Eq. (7), with $S_{H M}=-3 / 8 V(\phi)[10]$.

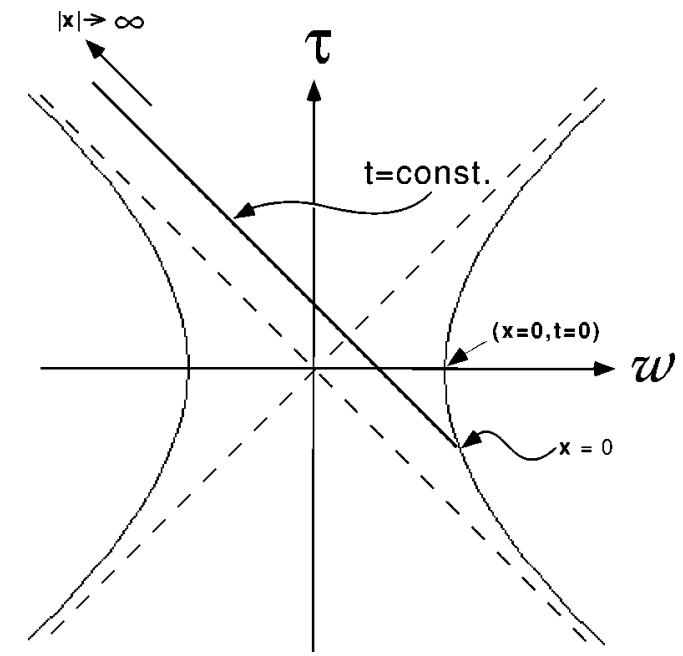

FIG. 6. De Sitter space can be viewed as a hyperboloid embedded in a 5-dimensional Minkowski space. Here we represent a section of this hyperboloid, along the plane $\tau, w$ in the embedding space. The region $w+\tau>0$ of the hyperboloid can be covered with flat FRW coordinates $\mathbf{x}, t$. A section $w+\tau=$ const corresponds to $t$ $=$ const.

In any case, we expect the rate to be nonzero in the general case. The reason is simply that the nucleation is not forbidden by any conservation laws, and thus should have a nonzero probability.

\section{FALSE VACUUM BUBBLES}

To study the geometry of false vacuum bubbles, we shall first assume that the bubble wall thickness is much smaller than all other relevant dimensions of the problem. The wall can then be treated as infinitely thin, and the spacetime regions on the two sides of the wall are de Sitter spaces of different vacuum energy. We now briefly review some properties of de Sitter space.

It is well known that de Sitter space can be pictured as a hyperboloid embedded in a flat 5-dimensional spacetime,

$$
\zeta^{2}+w^{2}-\tau^{2}=H^{-2} .
$$

where $\zeta=\left(\zeta^{1}, \zeta^{2}, \zeta^{3}\right)$ is a 3-vector. A section of the hyperboloid by the $w \tau$-plane is shown in Fig. 6 . The Euclideanized de Sitter space, which is used for constructing instantons, is obtained by analytic continuation $\tau=i \tau_{E}$,

$$
\zeta^{2}+w^{2}+\tau_{E}^{2}=H^{-2} .
$$

Geometrically, this is a 4-sphere of radius $H^{-1}$.

It will be convenient to use the Robertson-Walker flat coordinates in which the de Sitter metric takes its most familiar form,

$$
d s^{2}=d t^{2}-e^{2 H t} d \mathbf{x}^{2}
$$

These coordinates are related to the hyperboloid coordinates by

$$
\tau=H^{-1} \sinh (H t)+\frac{1}{2} H \mathbf{x}^{2} e^{H t}
$$




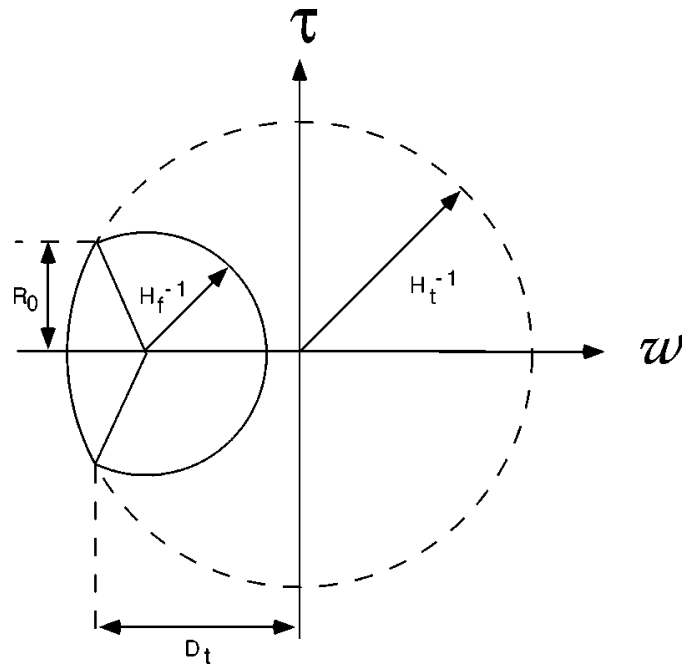

FIG. 7. The Coleman-de Luccia instanton for bubble nucleation can be obtained by matching two 4-spheres of radii $H_{f}^{-1}$ and $H_{t}^{-1}$. The two spheres join at a 3-sphere which represents the Euclideanized worldsheet of the domain wall.

$$
\begin{gathered}
w=H^{-1} \cosh (H t)-\frac{1}{2} H \mathbf{x}^{2} e^{H t}, \\
\zeta=\mathbf{x} e^{H t},
\end{gathered}
$$

which can be inverted to give

$$
t=H^{-1} \ln [H(w+\tau)], \quad \mathbf{x}=\frac{H^{-1} \zeta}{w+\tau} .
$$

Constant- $t$ surfaces are obtained as intersections of the hyperboloid with null hyperplanes $w+\tau=$ const (see Fig. 6). The surface $t=-\infty$ corresponds to $w+\tau=0$, and thus the coordinate system (10) covers only half of de Sitter space.

Let $H_{f}^{-1}$ and $H_{t}^{-1}$ be de Sitter horizons corresponding respectively to the false and true vacuum energy densities, $\rho_{f}$ and $\rho_{t}$. Clearly, $\rho_{t}<\rho_{f}$ and $H_{f}^{-1}<H_{t}^{-1}$. The thin wall approximation requires that the wall thickness be much smaller than $H_{f}^{-1}$. In this case, the Coleman-de Luccia instanton for bubble nucleation can be obtained by matching two 4spheres of radii $H_{f}^{-1}$ and $H_{t}^{-1}$ (see Fig. 7). The two spheres are joint at a 3-sphere which represents the Euclideanized worldsheet of the domain wall. Its radius $R_{0}$ is determined by $\rho_{f}, \rho_{t}$, and the wall tension $\sigma$ [33]. The 5-dimensional coordinates can always be chosen so that this worldsheet lies in a plane of constant $w$. In the figure it is $w=-D_{t}$, where

$$
D_{t}=\left(H_{t}^{-2}-R_{0}^{2}\right)^{1 / 2} \text {. }
$$

The Lorentzian evolution of the bubble is given by two hyperboloids similarly matched along a constant- $w$ plane (Fig. 8). Descriptions of both true and false vacuum bubbles can be obtained with an appropriate slicing of this spacetime by equal-time surfaces.

In the case of false vacuum bubbles, equal-time surfaces can be chosen to be the surfaces $w+\tau=$ const (Fig. 8). Then, each constant- $t$ slice consists of a spherical region of false vacuum embedded in an infinite, spatially-flat region of true vacuum. Since the spatial geometry of these slices is flat both

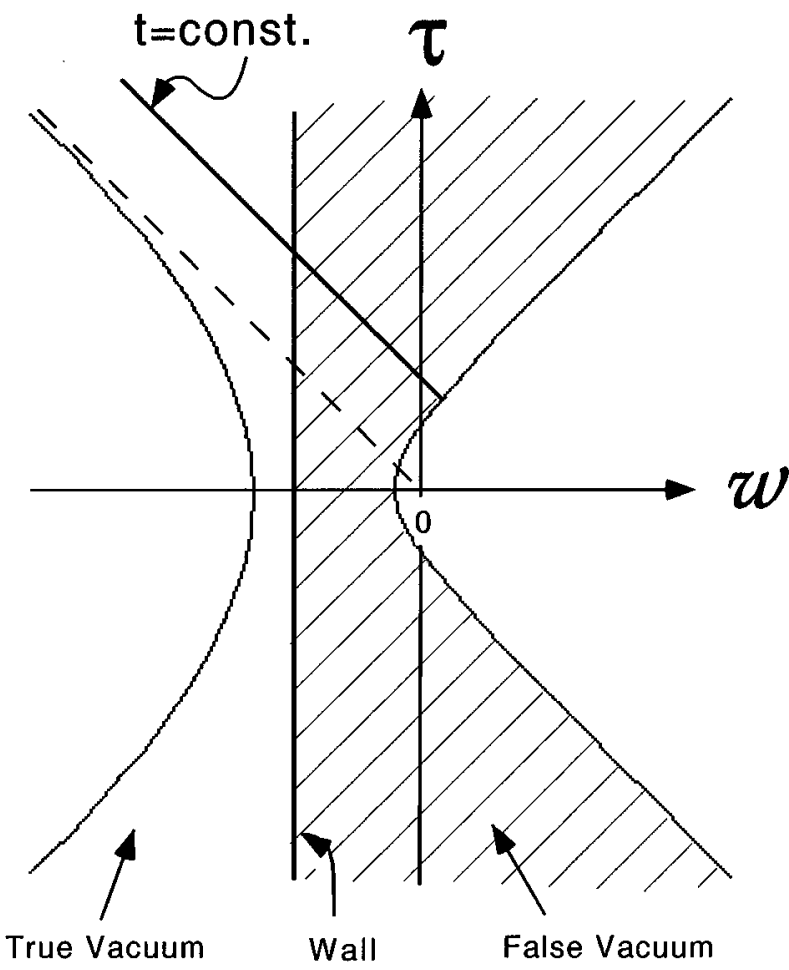

FIG. 8. The spacetime representing a false vacuum bubble in true vacuum.

inside and outside the bubble, the volume that is removed from true vacuum by the appearance of the bubble is equal to the volume of false vacuum which replaces it (this would not be the case if we used closed spatial sections, for instance). We can use the coordinates (10) with $H=H_{t}$ to describe the exterior true-vacuum region. The wall worldsheet is at $w=$ $-D_{t}$, and from Eq. (12) the radius of the bubble at time $t$ is

$$
R_{f}^{2}(t)=H_{t}^{-2}\left(e^{2 H_{t} t}+2 D_{t} H_{t} e^{H_{t} t}+1\right),
$$

where $R(t)=|\mathbf{x}(t)| e^{H_{t} t}$. We see that the radius approaches the horizon size $H_{t}^{-1}$ at $t \rightarrow-\infty$. The bubble wall accelerates in the direction of the false vacuum, so that its comoving radius $|\mathbf{x}(t)|$ is contracting, but the physical radius grows exponentially due to the expansion of the universe. A conformal diagram for the bubble spacetime is shown in Fig. 9.

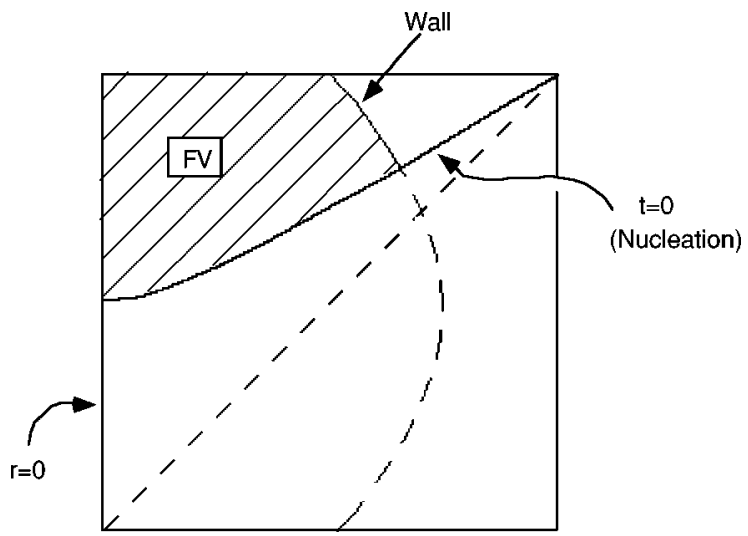

FIG. 9. Conformal diagram of the false vacuum bubble spacetime, for the Lee and Weinberg model. 
Since $R(t)$ is a monotonically growing function of time, there is no "bounce" moment at which one can say that the nucleation occurs. The situation here is similar to that for nucleation of topological defects in de Sitter space [29], and as in the latter case, we shall regard the bubble (16) as "formed" at $t \sim 0$, when its radius begins to grow exponentially.

For the trajectory given in Eq. (16), the region of true vacuum which has been removed and replaced by true vacuum was centered at the point $(t=0, \mathbf{x}=0)$ (see Figs. 6 and 8 ). We shall refer to this point as the center of symmetry of the bubble trajectory. (Notice that this point may not belong to the actual classical spacetime, because it is precisely in the region where bubble nucleation takes place, but it does belong to the five-dimensional embedding space.) Performing Lorentz transformations in the embedding space, we can obtain bubbles whose center of symmetry is at any time $t$ $=t_{0}$ and at any location. This gives

$$
R_{f}^{2}(t)=H_{t}^{-2}\left[e^{2 H_{t}\left(t-t_{0}\right)}+2 D_{t} H_{t} e^{H_{t}\left(t-t_{0}\right)}+1\right] .
$$

We can think of these as bubbles "formed" at $t \sim t_{0}$. The asymptotic behavior of the bubble radius at large times is

$$
R_{f}(t) \approx H_{t}^{-1} e^{H_{t}\left(t-t_{0}\right)}, \quad t-t_{0} \gg H_{t}^{-1} .
$$

To describe a true vacuum bubble in a false vacuum background, we choose equal-time surfaces to be $w-\tau=$ const. Using the coordinates (10) with $H=H_{f}$ to describe the exterior of the bubble, we find that the bubble radius at time $t$ is given by

$$
R_{t}^{2}(t)=H_{f}^{-2}\left(e^{2 H_{f} t}-2 D_{f} H_{f} e^{H_{f} t}+1\right),
$$

where

$$
D_{f}=\left(H_{f}^{-2}-R_{0}^{2}\right)^{1 / 2} .
$$

In contrast to the false-vacuum bubble case, the radius (19) has a minimum, $R_{\text {min }}=R_{0}$, at $t_{n}=H_{f}^{-1} \ln \left(D_{f} H_{f}\right)$, and we can regard this time as the moment of bubble nucleation. Equation (19) can then be rewritten as

$$
R_{t}^{2}(t)=D_{f}^{2}\left[e^{2 H_{f}\left(t-t_{n}\right)}-2 e^{H_{f}\left(t-t_{n}\right)}\right]+H_{f}^{-2},
$$

with the late-time behavior

$$
R_{t}(t) \approx D_{f} e^{H_{f}\left(t-t_{n}\right)}, \quad t-t_{n} \gg H_{f}^{-1} .
$$

Note, however that

$$
R_{t}(t) \approx H_{f}^{-1} e^{H_{f}\left(t-t_{0}\right)}, \quad t-t_{0} \gg H_{f}^{-1},
$$

where we denote by $t_{0}$ the location of the center of symmetry of the bubble wall as seen from the outside [see the discussion around Eq. (17)].

Our main interest in this paper is in models with $\rho_{t}$ $\ll \rho_{f}$. In such models, the radius of false vacuum bubbles is $R(t)>H_{t}^{-1} \gg H_{f}^{-1}$, and thus the thin wall approximation can be used to describe the bubble evolution even when the wall thickness is $\delta \sim H_{f}^{-1}$. (Note however that in this case the thin wall approximation breaks down for the instanton itself and for the early evolution of true vacuum bubbles.)

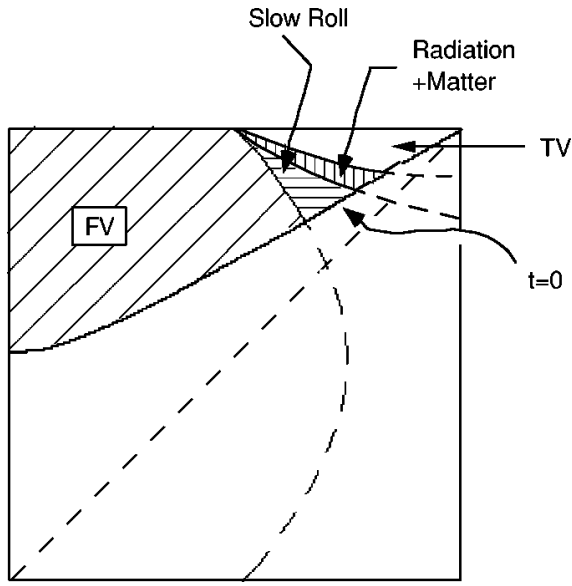

FIG. 10. Same as in Fig. 9 but for the case of a one field model of open inflation.

In a single-field model of open inflation, with a potential as in Fig. 3, the conformal diagram for a false vacuum bubble is shown in Fig. 10.

So far in this section we assumed that the true vacuum has a positive energy density. If the vacuum energy is in fact zero, then the horizon radius in thermalized regions keeps growing with time, and false vacuum bubbles eventually come within the horizon. The bubbles are then seen as black holes from the outside. The bubble nucleation in this case is similar to the quantum creation of baby universes, as discussed in Refs. [35,34,10]. Black holes eventually evaporate and baby universes pinch off.

\section{DID THE UNIVERSE HAVE A BEGINNING?}

Assuming that some rather general conditions are met, it was shown in Ref. [15] that inflationary models cannot be geodesically complete to the past, that is, they require some sort of a beginning. The assumptions that lead to this result are the following.

(A) The universe is causally simple [38,39].

(B) The universe is open.

(C) The null convergence condition [40]

(D) The finite past-volume difference condition.

The first two of these assumptions do not appear to be crucial for the proof, and extensions of the theorem have been obtained to some closed universes [16] and to some universes with a more complicated causal structure [17].

The null convergence condition is closely related to the weak energy condition, which requires that the energy density is non-negative when measured by any observer. Classically, this is satisfied by all known forms of matter, including a relativistic scalar field, but violations of the null convergence condition are possible as a result of quantum fluctuations. Such violations tend to occur in the inflating regions of spacetime whenever quantum fluctuations result in a local increase of the expansion rate, $d H / d t>0$ [36]. They are sufficient to invalidate the theorem in models characterized by a substantial variation of $V(\phi)$ in the range of $\phi$ where quantum fluctuations are non-negligible. This includes all models of "chaotic", inflation, but not some open and "new" inflationary models.

Turning now to the effects of recycling, we shall argue 
that they invalidate both assumptions (C) and (D), so that the theorem as it stands does not apply to any inflationary model. In the case of assumption (C), the reason is the same as before: quantum fluctuations from thermalized regions back to the inflating phase increase the expansion rate in the affected regions of space, and the null convergence condition is violated.

The finite past-volume difference condition (D) can be formulated as follows. Given a point $P$ in the inflating region and a point $Q$ to the past of $P$, consider the difference of their pasts. This is a spacetime region including all points to the past of $P$, but not of $Q$. The condition (D) requires that the spacetime volume of this region should be finite. The original motivation for this condition was based on the picture of eternally inflating universe without recycling, as illustrated in Fig. 1. It can be shown that thermalization surfaces, which separate inflating and thermalized regions of spacetime, are spacelike surfaces [15]. Therefore, if $Q$ is a point in an inflating region, then, disregarding recycling, all points in its past must also lie in the inflating region. For inflation to persist from $Q$ to $P$, no thermalized regions should be formed in the difference of the pasts of the two points. Now, it seems plausible that there is a zero probability for no thermalized regions to form in an infinite spacetime volume. Then it follows that condition (D) is necessary for inflation to persist in the future time direction. This condition is difficult to satisfy, since the spacetime region representing the difference of the pasts of the two points has an infinite extent along the past-directed null geodesics.

In a recycling universe, this logic does not apply, since points in inflating regions can have thermalized regions in their past. In this case, the spacetime region relevant for persistence of inflation between a pair of points is not the entire difference of their pasts, but only the part of this difference which lies in the same inflating region as the two points. In other words, it is the part of the difference of the pasts which is to the future of the "nucleation surface" (see Fig. 9). The volume of this region is obviously finite. Hence, assumption (D) is not suitable for a recycling universe.

In models with a vanishing true vacuum energy, only a small fraction of thermalized volume gets recycled. However, there still appears to be a possibility that the universe has a nested structure, with all inflating regions originating as quantum fluctuations inside thermalized regions.

We thus conclude that the theorems of Refs. [15-17] no longer apply when the recycling nature of the universe is taken into account. This may open the door for constructing nonsingular, steady-state inflationary models. We emphasize, however, that our analysis does not imply that such models do in fact exist. It has been argued in Refs. [37,36] that inflation, when continued to the past, is necessarily preceded by a period of contraction, as in the exact de Sitter space. During this period, the thermalized regions would merge, the density perturbations would grow very fast, and the universe would rapidly reach a grossly inhomogeneous state from which it is not likely to recover. The arguments in $[37,36]$ do not rely on weak energy or finite past-volume difference conditions, and may possibly be extended to the case of a recycling universe. These arguments, although suggestive, fall short of a proof, and the problem requires further investigation.

\section{STOCHASTIC FORMALISM}

A quantitative description of the recycling universe can be given using the formalism of stochastic inflation developed in Refs. $[2,8,19]$. A straightforward extension of this formalism will be required, and to introduce the necessary modifications, we shall first consider the Lee-Weinberg model [11] with a potential as in Fig. 2.

\section{A. Lee-Weinberg model}

Consider an ensemble of comoving observers whose world lines are orthogonal to some spacelike hypersurface $\Sigma$. Let $p_{f}(\tau)$ and $p_{t}(\tau)$ be the fractions of observers in false and true vacuum, respectively,

$$
p_{f}(\tau)+p_{t}(\tau)=1,
$$

where $\tau$ is the proper time along the observer's world lines measured from their intersection with $\Sigma$. The time evolution of $p_{f}$ and $p_{t}$ is described by the system of equations

$$
\begin{aligned}
& d p_{f} / d \tau=-\kappa_{f} p_{f}+\kappa_{t} p_{t}, \\
& d p_{t} / d \tau=-\kappa_{t} p_{t}+\kappa_{f} p_{f} .
\end{aligned}
$$

Here, $\kappa_{f}\left(\kappa_{t}\right)$ is the probability, per unit time, for an observer who is presently in the false (true) vacuum to find himself within a true (false) vacuum bubble.

From Eq. (21), we see that a false-vacuum observer will be affected only by bubbles nucleating within a sphere of radius $D_{f}$ centered on that observer. The bubbles take time $\sim H_{f}^{-1}$ to traverse this distance, but in the stochastic inflation formalism we shall be interested in quantities smeared over a spacetime scale $\sim H^{-1}$, so we shall disregard this time delay and write

$$
\kappa_{f} \approx \Gamma_{f}^{(n)} \frac{4 \pi}{3} D_{f}^{3}
$$

where $\Gamma_{f}^{(n)}$ is the rate of bubble nucleation per unit spacetime volume.

In an expanding universe, however, the rate of nucleation per unit spacetime volume has to be defined with some care. This is because this rate depends on what we choose as the nucleation time, which is not always sharply defined (especially when the size of bubbles becomes comparable to the Hubble radius). For instance, we can change our definition of nucleation time from $t_{n}$ to $t_{0}$, where $t_{n}$ is defined by Eq. (21) as the time at which the physical radius of the bubble reaches its minimum value $R_{0}$, and $t_{0}$ is defined as the center of symmetry of the bubble wall trajectory as seen from the outside (as explained in Sec. III). In changing the definition of nucleation time, we must simultaneously change the definition of nucleation rate per unit volume, because the physical volume has increased by the amount $\exp \left[3 H_{f}\left(t_{0}-t_{n}\right)\right]$ in the intervening time. Distinguishing by their superindex the rates associated with both choices of nucleation time, we have

$$
\Gamma_{f}^{(n)}=\Gamma_{f}^{(0)} \exp \left[3 H_{f}\left(t_{0}-t_{n}\right)\right]
$$

Hence, Eq. (27) can be rewritten as 


$$
\kappa_{f} \approx \Gamma_{f}^{(0)} \frac{4 \pi}{3} H_{f}^{-3}
$$

In the case of false vacuum bubbles the physical radius grows monotonically with time, so the analogue of $t_{n}$ does not exist. We shall adopt the convention identifying the nucleation time with $t_{0}$ in Eq. (17). Then the comoving region affected by the bubble is a sphere of radius $H_{t}^{-1}$, and we can write

$$
\kappa_{t} \approx \Gamma_{t}^{(0)} \frac{4 \pi}{3} H_{t}^{-3}
$$

where $\Gamma_{t}^{(0)}$ is the corresponding nucleation rate. We note that the radius of the affected region and the rate would both be modified with a different choice of nucleation time, while $\kappa_{t}$ would remain unchanged.

The solution of Eqs. (25),(26) is

$$
\begin{aligned}
& p_{f}(\tau)=p_{f}^{(0)}+A e^{-b \tau}, \\
& p_{t}(\tau)=p_{t}^{(0)}-A e^{-b \tau},
\end{aligned}
$$

where the constant $A$ is determined by the initial conditions, $b=\kappa_{f}+\kappa_{t}$, and $p_{f}^{(0)}, p_{t}^{(0)}$ is a stationary distribution defined by

$$
\begin{gathered}
\frac{p_{f}^{(0)}}{p_{t}^{(0)}}=\frac{\kappa_{t}}{\kappa_{f}}=\frac{H_{f}^{3}}{H_{t}^{3}} \frac{\Gamma_{t}^{(0)}}{\Gamma_{f}^{(0)}}, \\
p_{f}^{(0)}+p_{t}^{(0)}=1 .
\end{gathered}
$$

We see that, regardless of the initial conditions, the probability distribution rapidly approaches the stationary distribution (32).

Let us compare the distribution (32) with that discussed by Lee and Weinberg [11]. The distributions agree provided that the nucleation rates which appear in their expressions are taken as $\Gamma_{t / f}^{(0)}$. Lee and Weiberg also argue that the ratio (32) can be given to one loop order as

$$
\frac{\kappa_{t}}{\kappa_{f}}=\frac{H_{f}^{3}}{H_{t}^{3}} \exp \left[-(3 / 8)\left(\rho_{t}^{-1}-\rho_{f}^{-1}\right)\right] .
$$

The absence of determinantal prefactors in this expression is justified by the fact that the bounce solution for true and false vacuum decay are the same, hence the primed determinants corresponding to fluctuations around the bounce cancel out in the ratio of rates. The effect of determinants corresponding to fluctuations around the true and false vacuum background instantons is just to renormalize the values of the corresponding effective cosmological constants. Hence, in (34), the vacuum densities $\rho_{t / f}$ should be understood as their "one loop corrected' values.

In Ref. [13] the nucleation rates for true and false vacuum bubbles were studied in the case when the gravitational backreaction of the bubble is ignored, so that the background geometry was taken to be an exact de Sitter space with the same Hubble constant $H$ inside and outside the bubbles. It was found that the number distribution of true or false $(t / f)$ vacuum bubbles centered around the point $\left(t_{0}, \mathbf{x}\right)$ could be written as $d \mathcal{N}_{t / f}=\left|\lambda_{t / f}\right| \exp \left(3 H t_{0}\right) d^{3} \mathbf{x} d t_{0}$. Here $\lambda_{t / f}$ $=A \exp \left(-B_{t / f}\right)$, where $A$ is a primed determinant which is the same for true and false vacuum bubbles, and $B_{t / f}=S_{B}-S_{t / f}$ is the difference between the bounce action and the background Euclidean action. The expression for $d \mathcal{N}$ is proportional to the physical volume element at time $t_{0}$, given by $\exp \left(3 H t_{0}\right) d^{3} \mathbf{x}$, so we can identify $\left|\lambda_{t / f}\right|$ with the rates $\Gamma_{t / f}^{(0)}$ defined above. Therefore, we have $\left(\Gamma_{t}^{(0)} / \Gamma_{f}^{(0)}\right)=\exp \left[S_{t}-S_{f}\right]$, in agreement with Eqs. (32),(34).

We introduced $p_{f}$ and $p_{t}$ as fractions of comoving observers in false and true vacuum, respectively. When the stationary distribution (32) is reached, an alternative interpretation will also be useful. The world line of each observer will repeatedly cross between true and false vacuum regions, and the quantity $p_{f}^{(0)}\left(p_{t}^{(0)}\right)$ gives the fraction of proper time the observer spends in false (true) vacuum.

Instead of the proper time $\tau$, one can use some other time variable, $t$, along the observer's worldlines. A possible choice is

$$
d t=H^{\alpha}(\tau) d \tau
$$

with $\alpha=0$ corresponding to the proper time and $\alpha=1$ to the "scale factor time." For an arbitrary $\alpha$, the evolution equations still have the form (25),(26), with $\tau$ replaced by $t$ and

$$
\begin{gathered}
\kappa_{f}=\Gamma_{f}^{(0)} \frac{4 \pi}{3} H_{f}^{-\alpha-3}, \\
\kappa_{t}=\Gamma_{t}^{(0)} \frac{4 \pi}{3} H_{t}^{-\alpha-3} .
\end{gathered}
$$

The stationary solution now is

$$
\frac{p_{f}^{(\alpha)}}{p_{t}^{(\alpha)}}=\left(\frac{H_{f}}{H_{t}}\right)^{\alpha} \frac{p_{f}^{(0)}}{p_{t}^{(0)}} .
$$

The $\alpha$-dependence of (38) can be easily understood: $p_{f}\left(p_{t}\right)$ is proportional to the amount of time spent by a comoving observer in false (true) vacuum, and if the time variable is changed as in (35), the ratio $p_{f} / p_{t}$ is modified by a factor $\left(H_{f} / H_{t}\right)^{\alpha}$.

\section{B. A more realistic model}

Let us now consider a two-field model of the type (6), except we shall assume that the false vacuum at $\chi=0$ corresponds to a single point, rather than a flat direction, in the field space. This is the case, for example, in models where $\chi$ and $\phi$ represent the radial and angular parts of a single complex field, $\Phi=\chi e^{i \phi}$. We shall assume further that the effective potential for the field $\phi$ is of the "new" inflation type and has a slow roll region $\phi_{*}^{(1)}<\phi<\phi_{*}^{(2)}$. Finally, to simplify the discussion, we shall disregard the evolution between the end of slow roll and true vacuum domination. That is, we shall assume that when the field $\phi$ rolls down to $\phi_{*}^{(j)}$, it gets directly to the true minimum of the potential with energy density $\rho_{t}^{(j)}$, where $j=1,2$. We shall refer to $\phi_{*}^{(j)}$ as "thermalization points"' and to the corresponding minima of the potential as the first and the second true vacua, $T V(1)$ 
and $T V(2)$. (If $\rho_{t}^{(1)}=\rho_{t}^{(2)}$, then of course only one of these vacua is a truly true vacuum.) The false vacuum will be abbreviated as $F V$.

Once again we introduce an ensemble of comoving observers and define $p_{t 1}(t), p_{t 2}(t)$ and $p_{f}(t)$ to be the fractions of the observers in $T V(1), T V(2)$ and $F V$, respectively. We also define $\mathcal{P}(\phi, t) d \phi$ as the fraction of observers who are, at time $t$, located in slow roll regions with the inflaton field between $\phi$ and $\phi+d \phi$. We can now combine the analysis in the preceding subsection with the standard formalism of stochastic inflation to obtain the system of equations describing the evolution of our model:

$$
\begin{gathered}
\frac{\partial \mathcal{P}}{\partial t}=-\frac{\partial J}{\partial \phi}-\widetilde{\kappa}_{f}(\phi) \mathcal{P}+\kappa_{f}(\phi) p_{f} \\
\frac{d p_{t 1}}{d t}=-\kappa_{t 1} p_{t 1}+\kappa_{f 1} p_{f}-J_{1} \\
\frac{d p_{t 2}}{d t}=-\kappa_{t 2} p_{t 2}+\kappa_{f 2} p_{f}+J_{2} \\
\frac{d p_{f}}{d t}=-\left(\kappa_{f 1}+\kappa_{f 2}\right) p_{f}+\kappa_{t 1} p_{t 1}+\kappa_{t 2} p_{t 2}-p_{f} \int \kappa_{f}(\phi) d \phi \\
+\int \widetilde{\kappa}_{f}(\phi) \mathcal{P} d \phi
\end{gathered}
$$

Here, $\kappa_{f j}$ corresponds to tunneling from $F V$ to $T V(j), \kappa_{t j}$ to tunneling from $T V(j)$ to $F V$; they are given by Eqs. (36),(37) with an extra index $j$ added to the appropriate quantities. $\kappa_{f}(\phi) d \phi$ corresponds to tunneling from $F V$ to a value $\phi$ in the interval $d \phi$ in the slow roll region, and $\widetilde{\kappa}_{f}(\phi)$ to tunneling from a slow roll region with a given value of $\phi$ to $F V$. By analogy with $(36),(37)$ we can write

$$
\begin{gathered}
\kappa_{f}(\phi)=\Gamma_{f}^{(0)}(\phi) \frac{4 \pi}{3} H_{f}^{-\alpha-3}, \\
\widetilde{\kappa}_{f}(\phi)=\widetilde{\Gamma}_{f}^{(0)}(\phi) \frac{4 \pi}{3} H^{-\alpha-3}(\phi) .
\end{gathered}
$$

To simplify the equations, we have disregarded tunneling between $T V(1)$ and $T V(2)$ and between $T V(j)$ and the slow roll region. These effects can be easily included if necessary.

The current $J(\phi, t)$ in Eq. (39) is given by

$$
J(\phi, t)=-D^{1-\beta}(\phi) \frac{\partial}{\partial \phi}\left[D^{\beta}(\phi) \mathcal{P}(\phi, t)\right]+v(\phi) \mathcal{P}(\phi, t),
$$

where the first term on the right-hand side describes the "diffusion" of the field $\phi$ due to quantum fluctuations, with a diffusion coefficient

$$
D(\phi)=H^{3-\alpha}(\phi) / 8 \pi^{2},
$$

the second term describes the classical "drift" with velocity

$$
v(\phi)=-H^{-\alpha}(\phi) H^{\prime}(\phi) / 4 \pi
$$

and

$$
H^{2}(\phi)=8 \pi V(\phi) / 3 .
$$

The integration in Eq. (42) is from $\phi_{*}^{(1)}$ to $\phi_{*}^{(2)}$, and the quantities $J_{j}$ in Eqs. (40),(41) are defined as $J_{j}(t)$ $\equiv J\left(\phi_{*}^{(j)}, t\right)$. The normalization condition

$$
\int \mathcal{P} d \phi+p_{f}+p_{t 1}+p_{t 2}=1
$$

is preserved by the evolution equations (39)-(42).

The parameter $\beta$ in Eq. (45) for the current represents the factor-ordering ambiguity in the diffusion equation with a position-dependent diffusion coefficient. The choices $\beta$ $=1 / 2$ and $\beta=1$ are usually referred to as Stratonovich and Ito factor ordering, respectively.

The boundary conditions for Eq. (39) are

$$
\frac{\partial}{\partial \phi}\left[D^{\beta}(\phi) \mathcal{P}(\phi, t)\right]_{\phi=\phi_{*}^{(j)}}=0 .
$$

They ensure that, once the field $\phi$ rolls down to $\phi_{*}^{(j)}$, it does not diffuse back to the slow-roll regime, but rather stays in the true vacuum $T V(j)$ until it tunnels to $F V$.

The system of equations (39)-(42) can be written symbolically in the operator form

$$
\frac{d \mathbf{P}}{d t}=\mathbf{M P}
$$

where the "vector" $\mathbf{P}(t)$ is $\mathbf{P}=\left\{p_{f}, p_{t 1}, p_{t 2}, \mathcal{P}(\phi)\right\}$. With an appropriate discretization of $\phi$, this can be rewritten in the form of a "master equation":

$$
\frac{d P_{i}}{d t}=\sum_{j}\left(w_{i j} P_{j}-w_{j i} P_{i}\right) \equiv \sum_{j} M_{i j} P_{j}
$$

Each quantity $w_{i j}$ is positive and has the meaning of the transition rate from state $j$ to state $i$. The matrix $M_{i j}$ can be represented as

$$
M_{i j}=w_{i j}-\delta_{i j} \sum_{k} w_{k i}
$$

and has the properties

$$
\begin{gathered}
M_{i j} \geqslant 0 \quad(i \neq j), \\
\sum_{i} M_{i j}=0 .
\end{gathered}
$$

The latter property ensures the conservation of probability, $\sum_{i} \dot{P}_{i}=0$. It also indicates that the matrix $\mathbf{M}$ has a left eigenvector $\mathbf{Q}=\{1,1,1, \ldots\}$ with zero eigenvalue, $\mathbf{Q M}=0$. Since $\mathbf{M}$ and its transpose have the same eigenvalues, it follows that $\mathbf{M}$ should also have a right zero eigenvector,

$$
\mathbf{M P}_{\mathbf{0}}=0 \text {, }
$$

indicating that our system of equations has a stationary solution.

The familiar method of solving Eq. (52) using a decomposition in eigenvectors cannot, in general, be applied be- 
cause the matrix $\mathbf{M}$ is not generally symmetric. However, some properties of its solutions can be derived using only Eqs. (54),(55). We shall assume that the matrix $\mathbf{M}$ is irreducible (otherwise, the master equation (51) describes several independent processes which can each be described by a master equation with an irreducible matrix $\mathbf{M})$. Then it can be shown [41] that (i) the zero eigenvalue, $\gamma_{0}=0$, is nondegenerate, (ii) all components of the corresponding eigenvector $\mathbf{P}_{\mathbf{0}}$ are non-negative, (iii) all other eigenvalues of $\mathbf{M}$ satisfy $\operatorname{Re} \gamma_{n}<0$, and (iv) the asymptotic behavior of $\mathbf{P}(t)$ at late times is

$$
\mathbf{P}(t \rightarrow \infty)=\mathbf{P}_{0} .
$$

In other words, the stationary solution is unique, and all solutions approach this stationary solution at $t \rightarrow \infty$. Although these results have been rigorously derived only for a finite set of $P_{n}$, we shall assume that they are still valid in the continuum limit.

If all eigenvalues of $\mathbf{M}$ are nondegenerate, then $\mathbf{M}$ can be diagonalized, and the general solution of (51) can be written as

$$
\mathbf{P}(t)=\sum_{n=0}^{\infty} \mathbf{P}_{n} e^{\gamma_{n} t}
$$

where $\mathbf{P}_{n}$ are eigenvectors of the operator $\mathbf{M}$, and $\gamma_{n}$ are the corresponding eigenvalues,

$$
\mathbf{M P}_{n}=\gamma_{n} \mathbf{P}_{n}
$$

Since $\mathbf{M}$ is real, its eigenvalues and eigenvectors come in complex conjugate pairs. In the case of degenerate eigenvalues, the solution is more complicated [42].

In the absence of recycling, $\kappa_{t j}=\widetilde{\kappa}_{f}(\phi)=0$, and the stationary solution of the system (39)-(42) is trivial: $p_{t j}$ $=$ const, $p_{f}=\mathcal{P}(\phi)=0$. The standard analysis of stochastic inflation $[2,8,19]$ has been done for a slow-roll inflation without a metastable false vacuum. Then Eq. (39) reduces to a Fokker-Planck equation for $\mathcal{P}(\phi, t)$,

$$
\frac{\partial \mathcal{P}}{\partial t}=-\frac{\partial J}{\partial \phi} \equiv \mathcal{M P}
$$

It can be shown [43] that, with an appropriate choice of a scalar product, the differential operator $\mathcal{M}$ is Hermitian. Hence, all its eigenvalues are real and the eigenvectors form a complete orthonormal set. An eigenvector expansion of the form (58) is then always possible, and the asymptotic behavior of $\mathcal{P}(t)$ is

$$
\mathcal{P}(\phi, t \rightarrow \infty)=f(\phi) \exp \left(\gamma_{m} t\right) .
$$

Here, $\gamma_{m}<0$ is the largest eigenvalue of $\mathcal{M}$. In this standard approach the distribution $\mathcal{P}(\phi, t)$ is not normalized: the observers who left the slow roll range through the boundaries at $\phi_{*}^{(j)}$, never return, and $\int \mathcal{P} d \phi$ decreases exponentially with time.

\section{The fractal dimension}

In the case of "new" inflation without recycling, the inflating part of the universe represents a self-similar fractal of dimension $[4,21]$

$$
d=3+\gamma_{m} H_{m}^{\alpha-1} .
$$

Here, as in Eq. (61), $\gamma_{m}$ is the largest eigenvalue of the Fokker-Planck operator $\mathcal{M}$, and $H_{m}$ is the expansion rate at the maximum of $V(\phi)$ [44]. For a comoving sphere of radius $R$ centered on a point in the inflating region, the inflating volume within the sphere is $\mathcal{V}(R) \propto R^{d}$, which is a fraction

$$
f(R) \propto R^{d-3}
$$

of the total volume of the sphere. As the sphere expands, $R$ $\propto \exp \left(H_{m}^{1-\alpha} t\right)$, this fraction decreases as $f \propto \exp \left(\gamma_{m} t\right)$, and vanishes at $t \rightarrow \infty$. Hence, the inflating region represents a set of measure zero in the limit $t \rightarrow \infty$.

On the other hand, the inflating part of the volume in a recycling universe is constantly replenished by tunneling from the true vacuum. As a result, the inflating region occupies a non-vanishing fraction of the total volume, so that $\mathcal{V}(R) \propto R^{3}$ and $d=3$.

We note, however, that a recycling universe does contain fractal regions of dimension $d<3$. Take for example the Lee-Weinberg model of Sec. V A and consider a comoving volume which is initially filled with $F V$. What remains of this $F V$ in the limit $t \rightarrow \infty$ is a fractal of dimension [37]

$$
d_{f}=3-\Gamma_{f}^{(0)} \frac{4 \pi}{3} H_{f}^{-4} .
$$

All the remaining part of the volume is occupied by true vacuum bubbles, but what remains of the $T V$ inside the bubbles at $t \rightarrow \infty$ is also a fractal of dimension

$$
d_{t}=3-\Gamma_{t}^{(0)} \frac{4 \pi}{3} H_{t}^{-4} .
$$

The $F V$ bubbles inside each $T V$ bubble have dimension $d_{f}$, and they are in turn filled by $T V$ bubbles of dimension $d_{t}$. The fractal structure of realistic models is of course more complicated.

\section{Choosing the factor ordering}

One of the problems with interpreting the results of the stochastic inflation formalism is the dependence of these results on the choice of the time variable $t$ and on the factor ordering in the Fokker-Planck equation (39),(45) [19,27]. We have parametrized these choices by the parameters $\alpha$ and $\beta$. Now we are going to argue that there is a preferred choice of $\beta$ which allows at least a partial resolution of these problems.

As we discussed in Sec. IV A, the stationary distribution $\mathbf{P}_{0}$ gives the fraction of time spent by a comoving observer in false and true vacua and in different parts of the slow-roll range. This distribution should of course depend on how we define the time variable, but the dependence should be trivial: 


$$
\mathcal{P}(\phi) \propto H^{\alpha}(\phi), \quad p_{f} \propto H_{f}^{\alpha}, \quad p_{t j} \propto H_{t j}^{\alpha} .
$$

To ensure that this is indeed the case, we can require that, in the stationary version of Eq. (51),

$$
\mathbf{M P}=0 \text {, }
$$

all components of $\mathbf{P}$ appear only in combinations $H^{-\alpha}(\phi) \mathcal{P}(\phi), H_{f}^{-\alpha} p_{f}, H_{t j}^{-\alpha} p_{t j}$, and that there is no other dependence on $\alpha$. This fixes

$$
\beta=1,
$$

which corresponds to Ito factor ordering.

It should be noted that the family of factor ordering choices parametrized by $\beta$ in Eq. (45) does not include all possibilities. Although our requirement (66) is sufficient to fix $\beta$ uniquely, it does not determine a unique factor ordering in the general case. For example, we could replace the diffusion term in (45) by

$$
\begin{aligned}
D^{1-\beta}(\phi) \frac{\partial}{\partial \phi} & {\left[D^{\beta}(\phi) \mathcal{P}(\phi, t)\right] } \\
& \rightarrow h^{-1}(\phi) \frac{\partial}{\partial \phi}[h(\phi) D(\phi) \mathcal{P}(\phi, t)] .
\end{aligned}
$$

The condition (66) is satisfied for an arbitrary, $\alpha$-independent function $h(\phi)$.

\section{E. Some solutions}

The system of equations (39)-(42) looks rather intimidating, but stationary solutions of this system can actually be found in some special cases.

Let us first assume that tunneling from slow roll to false vacuum can be neglected, that is, $\widetilde{\kappa}_{f}(\phi)=0$. This means that the evolution proceeds along the path $F V \rightarrow$ slow roll $\rightarrow T V \rightarrow F V \rightarrow \ldots$, possibly with occasional tunneling directly from $F V$ to $T V$. Then, with $\beta=1$, the stationary version of (39) can be written as

$$
\partial_{\phi} J(\phi)=\kappa(\phi) p_{f},
$$

where

$$
J(\phi)=-\partial_{\phi}[D(\phi) \mathcal{P}(\phi)]+v(\phi) \mathcal{P}(\phi) .
$$

This is easily integrated to give

$$
\begin{aligned}
\mathcal{P}(\phi)= & -\frac{8 \pi^{2} p_{f}}{H^{3-\alpha}(\phi)} e^{\pi / H^{2}(\phi)} \\
& \times\left[\int_{\phi_{*}^{(1)}}^{\phi} d \phi^{\prime} e^{-\pi / H^{2}\left(\phi^{\prime}\right) \int_{\phi_{*}^{(1)}}^{\phi^{\prime}} d \phi^{\prime \prime} \kappa_{f}\left(\phi^{\prime \prime}\right)}\right. \\
& \left.+C_{1} \int_{\phi_{*}^{(1)}}^{\phi} d \phi^{\prime} e^{-\pi / H^{2}\left(\phi^{\prime}\right)}+C_{2}\right],
\end{aligned}
$$

where we have used the expressions $(46),(47)$ for $D(\phi)$ and $v(\phi)$.

The integration constants $C_{1}, C_{2}$ can now be found from the boundary conditions (50) (with $\beta=1$ ). Thus we obtain the distribution $\mathcal{P}(\phi)$ in terms of $p_{f}$. Evaluating $J_{j}$ $=J\left(\phi_{*}^{(j)}\right)$ and substituting in Eqs. (40),(41), we find $p_{t j}$ in terms of $p_{f}$. Finally, $p_{f}$ is found from the normalization condition (49). [Note that $p_{f}$ cannot be found from Eq. (42) which is a linear combination of the preceding three equations (39)-(41)]. The resulting expressions are rather cumbersome and we shall not reproduce them here.

As another example, we take a potential $V(\phi)$ of the form considered in Ref. [21], which consists of a flat portion where $H(\phi)=$ const, $\kappa_{f}(\phi)=$ const, $\tilde{\kappa}_{f}(\phi)=$ const, surrounded by two regions with a relatively large slope where the diffusion term is negligible. In the flat range of $\phi$, the Fokker-Planck equation is trivially solved. In the diffusionless regions,

$$
\partial_{\phi}[v(\phi) \mathcal{P}(\phi)]+\widetilde{\kappa}_{f}(\phi) \mathcal{P}(\phi)=\kappa_{f}(\phi) p_{f},
$$

and a straightforward integration gives

$$
\begin{aligned}
\mathcal{P}(\phi)= & -4 \pi p_{f} \frac{H^{\alpha}(\phi)}{H^{\prime}(\phi)} e^{-g(\phi)} \\
& \times\left[\int_{\phi_{*}^{(1)}}^{\phi} d \phi^{\prime} \kappa_{f}\left(\phi^{\prime}\right) e^{g\left(\phi^{\prime}\right)}+C_{1}\right],
\end{aligned}
$$

where

$$
g(\phi)=\int_{\phi_{*}^{(1)}}^{\phi} d \phi^{\prime} \frac{\widetilde{\kappa}_{f}\left(\phi^{\prime}\right)}{v\left(\phi^{\prime}\right)}
$$

for the region bounded by $\phi_{*}^{(1)}$, and similarly for the second region bounded by $\phi_{*}^{(2)}$. The integration constants and the values of $p_{f}$ and $p_{t j}$ can be determined by matching the solutions at the boundaries between the flat and diffusionless regions and by using Eqs. (40),(41) and the normalization condition (49).

In the general case, the solution (74) should still apply in the range of $\phi$ sufficiently close to the thermalization points, where diffusion is negligible. If tunneling between this range and the false vacuum is unimportant, then the solution takes a particularly simple form,

$$
\mathcal{P}(\phi)=-4 \pi C_{1} p_{f} \frac{H^{\alpha}(\phi)}{H^{\prime}(\phi)},
$$

and similarly for the range of $\phi$ near $\phi_{*}^{(2)}$. The constants $C_{1}$ and $C_{2}$ can be determined only after solving the equation in the entire range of $\phi$.

The distribution (76) in the diffusionless range of $\phi$ should be compared with the corresponding distribution in the absence of recyclings [21],

$$
\mathcal{P}(\phi)=C \frac{H^{\alpha}(\phi)}{H^{\prime}(\phi)} \exp \left[-4 \pi \gamma_{m} \int_{\phi_{*}^{(1)}}^{\phi} d \phi^{\prime} \frac{H^{\alpha}\left(\phi^{\prime}\right)}{H^{\prime}\left(\phi^{\prime}\right)}\right] \text {. }
$$

This can be drastically different from (76) even if the tunneling probabilities are very small. Hence, there is no continuous transition between recycling and no-recycling regimes in the limit of vanishing tunneling probabilities. 


\section{F. Physical volume distribution}

The function $\mathbf{P}(t)$ characterizes the distribution of comoving volume between false and true vacua and different values of $\phi$ in the slow roll regime. One can introduce a similar function for the physical volume distribution [45-47]:

$$
\widetilde{\mathbf{P}}(t)=\left\{\widetilde{\mathcal{P}}(\phi, t), \tilde{p}_{f}(t), \tilde{p}_{t 1}(t), \tilde{p}_{t 2}(t)\right\} .
$$

It is defined so that $\widetilde{\mathcal{P}}(\phi, t) d \phi$ is the physical volume occupied by slow roll regions with $\phi$ in the range $d \phi$ at time $t$, etc. The distribution $\widetilde{\mathbf{P}}(t)$ satisfies a modified master equation,

$$
\frac{d \widetilde{\mathbf{P}}}{d t}=\mathbf{M} \widetilde{\mathbf{P}}+3 \mathbf{H}^{1-\alpha} \widetilde{\mathbf{P}} \equiv \widetilde{\mathbf{M}} \widetilde{\mathbf{P}}
$$

where the operator $\mathbf{H}^{1-\alpha}$ is given by

$$
\mathbf{H}^{1-\alpha}=\operatorname{diag}\left\{H^{1-\alpha}(\phi), H_{f}^{1-\alpha}, H_{t 1}^{1-\alpha}, H_{t 2}^{1-\alpha}\right\} .
$$

In an infinite universe, the volume is of course infinite, but the distribution $\widetilde{\mathbf{P}}(t)$ can be defined on a fixed comoving part of the universe. The form of the distribution at large $t$ is independent of the choice of the comoving region.

In the discretized version of Eq. (79), the matrix $\widetilde{\mathbf{M}}$ does not have the property (55), and the standard theorems for the master equation do not apply. However, the following statements can still be proved [48] using the Perron-Frobenius theorem about non-negative matrices: (i) $\widetilde{\mathbf{M}}$ has a real eigenvalue $\tilde{\gamma}_{0}$ which is greater than the real parts of all other eigenvalues and which is bounded by

$$
3 H_{\min }^{1-\alpha} \leqslant \widetilde{\gamma}_{0} \leqslant 3 H_{\max }^{1-\alpha},
$$

where $H_{\max }$ and $H_{\min }$ are respectively the largest and the smallest values of $H$; (ii) the corresponding eigenvector $\widetilde{\mathbf{P}}_{0}$ has non-negative components; (iii) $\widetilde{\gamma}_{0}$ is nondegenerate if $\widetilde{\mathbf{M}}$ is irreducible. The late-time asymptotic behavior of $\widetilde{\mathbf{P}}(t)$ is

$$
\widetilde{\mathbf{P}}(t \rightarrow \infty)=\widetilde{\mathbf{P}}_{0} e^{\tilde{\gamma}_{0} t} .
$$

In contrast to the comoving distribution $\mathbf{P}(t)$, the physical volume distribution $\widetilde{\mathbf{P}}(t)$ has a sensitive dependence on the choice of the time parameter $\alpha$, which does not reduce to the trivial form (66) $[19,21,27]$. The equation (79) for $\widetilde{\mathbf{P}}(t)$ is simplified if we choose the scale factor time, $\alpha=1$. In this case, $\mathbf{H}^{1-\alpha}=1$, and the solutions of (79) and (51) are related by $[19]$ :

$$
\widetilde{\mathbf{P}}_{\alpha=1}(t)=e^{3 t} \mathbf{P}_{\alpha=1}(t) .
$$

\section{G. Discussion}

The main conclusion of our analysis in this section is that the distribution of comoving observers in a recycling universe rapidly approaches a stationary form. This asymptotic distribution can be obtained as the eigenvector of the "master' operator $\mathbf{M}$ with a zero eigenvalue, $\gamma_{0}=0$ :

$$
\mathbf{M P} \mathbf{P}_{0}=0 \text {. }
$$

The formalism we developed here can be straightforwardly extended to include radiation and matter dominated periods between thermalization and true vacuum domination. One expects to find that the asymptotic distribution will still be stationary, with fixed fractions of comoving volume occupied by radiation and matter-dominated regions.

The picture in which comoving "observers" move in an endless cycle between $F V$, slow roll, matter domination, and $T V$, may be oversimplified. It should be understood, of course, that no material observer is likely to survive the transition between $T V$ and $F V$. Even if we think of an "observer' as an indestructible test particle, there seems to be no unique way to continue his world line into a nucleating bubble, since the surface at which we glue the bottom of the false vacuum bubble onto the true vacuum can be chosen in different ways. So we should probably think of our "observers" as being smeared over a horizon-size volume.

Next, we note that density fluctuations produced during inflation (or generated by topological defects) result in the formation of bound objects during the matter-dominated era. Some of these objects collapse to black holes, and observers in matter-dominated regions have a finite probability (per unit time) to end their world lines at black hole singularities. However, black holes eventually evaporate, giving back their volume to the true vacuum. Hence this effect would not alter our conclusions. The same happens with black holes that may spontaneously nucleate in false or true vacuum [49]. The rate of black hole pair production in true vacuum is proportional to $\exp \left[-1 / 8 \rho_{t}\right]$. This rate is considerably larger than that for nucleation of a false vacuum bubble, which in the case $\rho_{t} \ll \rho_{f}$ is proportional $\exp \left[-3 / 8 \rho_{t}\right]$. It is also possible that nucleated black holes may act as seeds for false vacuum bubble nucleation, as they do for true vacuum bubbles [50].

Finally, the inflaton potential $V(\phi)$ can be of the "chaotic' inflation type, with the slow roll range of $\phi$ extending to Planckian energy densities. Then there is a finite probability for an observer to get into this Planckian domain, where the classical description of spacetime breaks down. In the stochastic inflation formalism, this is accounted for by introducing a "Planck boundary" at some $\phi=\phi_{p}$, such that $V\left(\phi_{p}\right) \sim 1$. The loss of observers through the Planck boundary will generally result in $\gamma_{0}>0$ and $d<3$.

The same phenomenon of loss of observers will also occur if some of the minima of the potential have vanishing or negative cosmological constant. Once some comoving volume falls into one of these vacua it has no chance of being recycled.

\section{PROBLEMS WITH PREDICTIONS}

Different thermalized regions of the universe are generally characterized by different values of the constants of $\mathrm{Na}-$ ture and of the cosmological parameters (such as the density parameter $\Omega$ ). In the model that we used as an example in Sec. V, the universe can thermalize into two types of vacua, $T V 1$ and $T V 2$, and thus we have two possible sets of constants of Nature. The number of possibilities can, in principle, be much larger, and in some models the "constants", can even take values in a continuous range (examples are the effective gravitational constant in a Brans-Dicke-type theory 
[18] and the density parameter in some models of "open" inflation [6]). An intriguing question is whether or not we can "predict" which set of the constants we are most likely to observe.

One can try to determine the probability distribution for the constants with the aid of the "principle of mediocrity" which asserts that we are "typical" among the civilizations inhabiting the universe $[20,24,25,18]$. Here, the universe is understood as the entire spacetime; our civilization is assumed to be typical among all civilizations, including those that no longer exist and those that will appear in the future. The probability for us to observe a given set of constants is then proportional to the total number of civilizations in the corresponding type of thermalized regions. This number can be represented as the number of galaxies (which one can hope to estimate) times the number of civilizations per galaxy (which is left undetermined until the evolution of life and consciousness are better understood). Some of the constants, such as the cosmological constant or the density parameter, are not expected to affect the chances for a civilization to develop in a given galaxy, so one can hope to determine the probability distribution for such constants without any biological input.

In the case of a closed universe and finite (noneternal) inflation, this prescription for calculating probabilities is unambiguous. If the universe is spatially infinite, one can simply use the prescription for a fixed (sufficiently large) comoving volume. However, in an eternally inflating universe the spacetime volume and the number of civilizations are infinite, even for a region of a finite comoving size. One can deal with this problem by simply introducing a time cutoff and counting only the number of civilizations $N_{j}\left(t_{c}\right)$ that appeared prior to some moment of time $t_{c}$. Here, the index $j$ refers to the type of thermalized region. The ratio of probabilities can then be defined as the limit

$$
\frac{p_{1}}{p_{2}}=\lim _{t_{c} \rightarrow \infty} \frac{N_{1}\left(t_{c}\right)}{N_{2}\left(t_{c}\right)} .
$$

One finds, however, that the resulting probability distribution is extremely sensitive to the choice of the time variable $t$ $[51,19]$. This gauge-dependence casts doubt on any conclusions reached using this approach.

An alternative procedure, suggested in [21], is to introduce a cutoff at the time $t_{c}^{(j)}$, when all but a small fraction $\epsilon$ of the comoving volume destined to thermalize into regions of type $j$ has thermalized. The value of $\epsilon$ is taken to be the same for all types of thermalized regions, but the corresponding cutoff times $t_{c}^{(j)}$ are generally different. The limit $\epsilon \rightarrow 0$ is taken after calculating the probability distribution for the constants. It was shown in $[21,27]$ that the resulting probabilities are essentially insensitive to the choice of time parametrization. However, the same problem appears in a different guise. Linde and Mezhlumian [28] have found a family of gauge-invariant cutoff procedures parametrized by a dimensionless parameter $q$, with $q=0$ corresponding to the $\epsilon$-procedure described above. This indicates that the invariance requirement alone is not sufficient to define the probabilities uniquely. Some additional requirements that can fix the parameter $q$ have been discussed in [27].
Now, recycling introduces one more difficulty. In the absence of recycling, comoving regions could be uniquely characterized by the type of thermalized region they will evolve into. But in a recycling universe each comoving region goes through an endless succession of different types of thermalization. Hence, the $\epsilon$-procedure cannot be implemented in its present form [52].

In the face of these difficulties, one could look for entirely different approaches to defining the probabilities. One possibility is to abandon the requirement of gauge-invariance and assert that there is a preferred choice of the time variable $t$. If this approach is taken, then there is, arguably, a good reason to take the scale-factor time, $t=\ln a$, as the preferred choice $[8,53]$. The only variables that can be used as clocks in an inflating universe are the inflaton field $\phi$ and the scale factor $a$. The main requirement for a clock is a predictable classical behavior. In the range of $\phi$ where quantum fluctuations are important, $\phi$ is not suitable for this role, and the only remaining variable to be used as a clock is $a$.

Another possibility is to abandon the principle of mediocrity and invoke the ideal observers that we used to define the distribution $\mathbf{P}(t)$, rather than physical observers, to calculate probabilities. In a recycling universe, the worldline of an ideal observer crosses an infinite number of inflating and thermalized regions. The probabilities for different types of thermalized regions can then be defined as relative frequencies at which these regions are encountered along the worldline. This definition is obviously gauge-invariant. In the model of Sec. V B, it gives

$$
p_{1} / p_{2}=\left|J_{1} / J_{2}\right| .
$$

The gauge-invariance of (86) is easily verified from Eqs. (45),(47),(50),(66). In this approach, the most probable thermalized regions may turn out to be unsuitable for life, but this can be easily fixed by defining appropriate conditional probabilities.

As mentioned at the end of Sec. III, recycling may not be complete in models where there is a "Planck boundary" in the diffusion regime or where some of the minima of the effective potential have vanishing or negative effective cosmological constant. In this case, the worldlines of all ideal observers (except a set of measure zero) have a finite length, and a natural extension of Eq. (86) is

$$
\frac{p_{1}}{p_{2}}=\frac{\int_{0}^{\infty} d t\left|J_{1}(t)\right|}{\int_{0}^{\infty} d t\left|J_{2}(t)\right|} .
$$

This defines the probabilities as being proportional to the total number of encounters for a given type of region, averaged over all observers. The result depends on the initial distribution $\mathbf{P}(0)$ at $t=0$. Assuming that in this type of models the universe must have a beginning, and that it can be described by quantum cosmology, this initial distribution can be determined from the wave function of the universe.

Although the definition of probabilities in this approach is gauge-invariant, it is not quite satisfactory. The ideal observers have very little to do with real physical observers, and it is hard to justify why the likelihood of various observations made by our civilization should be related to an ensemble of such ideal observers. 
We have to conclude that, despite some effort, none of the approaches suggested so far appears to be particularly compelling. It may turn out that, after all, an eternally inflating universe does not admit a uniquely defined probability distribution for the constants of nature. If so, this does not necessarily mean that all possible sets of constants consistent with our existence are equally likely. Although the ratio $p_{1} / p_{2}$ may depend on the choice of cutoff procedure, it is conceivable that in some cases $p_{1} / p_{2} \gg 1$ for all reasonable choices. We would then "predict" that 1 is much more likely than 2 . It is possible that we will have to restrict ourselves to such "stable", predictions, which are insensitive to the choice of cutoff [54].

\section{CONCLUSIONS}

We have shown that the picture of the superlarge-scale structure of the inflationary universe is significantly modified by quantum fluctuations which bring parts of already thermalized regions back to the false vacuum, a process which we call recycling.

In particular, the question of whether or not the Universe had a beginning is reopened. Ignoring recycling, and under certain rather general conditions, it has been shown in Refs. $[37,15]$ that inflationary models require a beginning in time. In the recycling picture, this conclusion does not apply because it is only necessary that any false vacuum region has a beginning in time. One can therefore imagine a nested structure where all false vacuum regions are just bubbles which nucleated inside preexisting true vacuum bubbles, which in turn nucleated inside false vacuum bubbles and so on. Whether or not this pattern can be continued to the infinite past is an interesting open question.

We have extended the standard formalism of stochastic inflation $[2,8,19]$ to study the probability distribution of phases in which comoving observers (or comoving volume) find themselves in the recycling universe. Instead of the Fokker-Planck equation, one now has a more general master equation. We found that, in the case of complete recycling, all solutions of this equation rapidly approach a stationary form. This is in contrast to the standard case, where the probability distribution decreases exponentially with time, due to the loss of comoving volume at thermalization.

In the absence of recycling, the fractal dimension of the false vacuum comoving volume at large times is lower than three. Including recycling, this dimension is $d=3$ because comoving volume in false vacuum is continually replenished by nucleating false vacuum bubbles. The universe ends up in a highly convoluted state, where the fractal dimension of any connected false or true vacuum region is lower than three.

Finally, we have considered the question of "making predictions" for the constants of nature in the context of a recycling universe. The principle of mediocrity has been invoked in the past in order to obtain probability distributions for the constants. For the case of finite inflation the procedure is unambiguous: the probability is proportional to the number of civilizations that observed a given set of constants in the entire history of the universe. In the case of eternal inflation, the principle is not so easy to implement, because the number of thermalized regions with given values of the constants is infinite (even in a finite comoving region), and one has to introduce a regulator. If one simply counts all civilizations below some cutoff time, then the result depends strongly on the choice of time variable [19]. A gauge invariant cutoff prescription (i.e., one which does not depend on the time variable) was introduced in [21], but this prescription is not unique [28]. To make matters more complicated, the methods discussed so far cannot be directly applied to a recycling universe. We have considered some generalizations and alternative approaches, but none of them is particularly compelling.

Therefore, it seems that while the principle of mediocrity may offer some valid guidance, it may not be sufficient to unambiguously determine the probability distribution for the constants of nature. If this turns out to be the final answer, then "predictions" would only be possible in those cases when all reasonable cutoff methods or implementations of the principle yield a similar answer.

\section{ACKNOWLEDGMENTS}

We are grateful to Julie Traugut for suggestive conversations, to Serge Winitzki for very helpful discussions and to Andrei Linde for his comments. This work was partially supported by NATO under grant CRG 951301. J.G. acknowledges support from CICYT under contract AEN95-0882 and from European Project CI1-CT94-0004. The work of A.V. was supported in part by the National Science Foundation.
[1] For reviews see, for example, S. K. Blau and A. H. Guth, in 300 Years of Gravitation, edited by S. W. Hawking and W. Israel (Cambridge University Press, Cambridge, UK, 1978); A. D. Linde, Particle Physics and Inflationary Cosmology (Harwood Academic, Chur, Switzerland, 1990); E. W. Kolb and M. S. Turner, The Early Universe (Addison-Wesley, New York, 1990).

[2] A. Vilenkin, Phys. Rev. D 27, 2848 (1983).

[3] A. D. Linde, Phys. Lett. B 175, 395 (1986).

[4] M. Aryal and A. Vilenkin, Phys. Lett. B 199, 351 (1987).

[5] J. R. Gott, Nature (London) 295, 304 (1982); M. Sasaki, T. Tanaka, K. Yamamoto, and J. Yokoyama, Phys. Lett. B 317, 510 (1993); K. Yamamoto, M. Sasaki, and T. Tanaka, Astro- phys. J. 455, 412 (1995); M. Bucher, A. Goldhaber, and N. Turok, in Trends in Astroparticle Physics, Proceedings of the Workshop, Stockholm, Sweden, 1994, edited by L. Bergstrom et al. [Nucl. Phys. B (Proc. Suppl.) 43, 173 (1995)]; Phys. Rev. D 52, 3314 (1995).

[6] A. Linde and A. Mezhlumian, Phys. Rev. D 52, 6789 (1995).

[7] S. W. Hawking and I. Moss, Nucl. Phys. B224, 180 (1983).

[8] A. A. Starobinsky, in Current Topics in Field Theory, Quantum Gravity and Strings, edited by H. J. de Vega and N. Sanchez, Lecture Notes in Physics (Springer, Heidelberg, 1986).

[9] A. S. Goncharov and A. D. Linde, Sov. J. Part. Nucl. 17, 369 (1986). 
[10] A. Linde, Nucl. Phys. B372, 421 (1992).

[11] K. Lee and E. J. Weinberg, Phys. Rev. D 36, 1088 (1987).

[12] S. Coleman and F. De Luccia, Phys. Rev. D 21, 3305 (1980).

[13] J. Garriga, Phys. Rev. D 49, 6327 (1994); 49, 6343 (1994).

[14] A. Linde, in The Very Early Universe, edited by G. W. Gibbons and S. W. Hawking (Cambridge University Press, Cambridge, UK, 1983).

[15] A. Borde and A. Vilenkin, Phys. Rev. Lett. 72, 3305 (1994).

[16] A. Borde, Phys. Rev. D 50, 3692 (1994).

[17] A. Borde and A. Vilenkin, Int. J. Mod. Phys. D 5, 813 (1996).

[18] J. Garcia-Bellido and A. Linde, Phys. Rev. D 51, 429 (1995); J. Garcia-Bellido, A. Linde, and D. Linde, ibid. 50, 730 (1994).

[19] A. Linde, D. Linde, and A. Mezhlumian, Phys. Rev. D 49, 1783 (1994).

[20] A. Vilenkin, Phys. Rev. Lett. 74, 846 (1995).

[21] A. Vilenkin, Phys. Rev. D 52, 3365 (1995).

[22] A. Vilenkin and S. Winitzki, Phys. Rev. D 55, 548 (1997).

[23] H. Martel, P. R. Shapiro, and S. Weinberg, astro-ph/9701099.

[24] The principle of mediocrity is a version of the anthropic principle. For a review of the latter, see e.g. B. Carter, Philos. Trans. R. Soc. London A310, 347 (1983); J. D. Barrow and F. J. Tipler, The Antropic Cosmological Principle (Clarendon Press, Oxford, 1986). Ideas related to the principle of mediocrity have also been discussed by Albrecht [25], Garcia-Bellido and Linde [18], and Tegmark [26].

[25] A. Albrecht, in The Birth of the Universe and Fundamental Forces, edited by F. Occhionero (Springer-Verlag, Berlin, 1995).

[26] M. Tegmark, gr-qc/9704009 (unpublished).

[27] S. Winitzki and A. Vilenkin, Phys. Rev. D 53, 4298 (1996).

[28] A. Linde and A. Mezhlumian, Phys. Rev. D 53, 4267 (1996).

[29] R. Basu and A. Vilenkin, Phys. Rev. D 46, 2345 (1992); 50, 7150 (1994).

[30] K. Lee and E. Weinberg, Nucl. Phys. B267, 181 (1986); M. J. Duncan and L. G. Jensen, Phys. Lett. B 291, 109 (1992); S. Dimopoulos, G. Dvali, R. Rattazzi, and G. Giudice, hep-ph/9705307.

[31] L. Jensen and P. Steinhardt, Nucl. Phys. B317, 693 (1989).

[32] T. S. Bunch and P. C. W. Davies, Proc. R. Soc. London A360, 117 (1978); A. Vilenkin and L. Ford, Phys. Rev. D 26, 1231 (1982).

[33] V. A. Berezin, V. A. Kuzmin, and I. Tkachev, Phys. Lett. 120B, 91 (1983); S. Parke, ibid. 121B, 313 (1983).

[34] W. Fishler, D. Morgan, and J. Polchinski, Phys. Rev. D 41, 2638 (1990).

[35] E. Farhi and A. H. Guth, Phys. Lett. B 183, 149 (1987); S. K.
Blau, E. I. Guendelman, and A. H. Guth, Phys. Rev. D 35, 1747 (1987).

[36] A. Borde and A. Vilenkin, Phys. Rev. D 56, 717 (1997).

[37] A. Vilenkin, Phys. Rev. D 46, 2355 (1992).

[38] This is the requirement that the spacetime have a simple causal structure. In particular, it excludes complicated topological interconnections between different regions of spacetime. See $[15,39]$ for a precise discussion and a diagram.

[39] A. Borde and A. Vilenkin, in Relativistic Astrophysics: The Proceedings of the Eighth Yukawa Symposium, edited by M. Sasaki (Universal Academy Press, Japan, 1995).

[40] This requires that there exist certain pairs of points such that the spacetime volume of the difference of their pasts is finite. See $[15,39]$.

[41] N. G. van Kampen, Stochastic Processes in Physics and Chemistry (North-Holland, Amsterdam, 1981).

[42] F. R. Moulton, Differential Equations (Dover, New York, 1958).

[43] H. Risken, The Fokker-Planck Equation (Springer-Verlag, Berlin, 1989).

[44] The fractal dimension in (62) appears to depend on the choice of the parameter $\alpha$ (that is, on the time parametrization). However, it has been shown in [27] that the product $\gamma_{m} H_{m}^{\alpha-1}$ is essentially independent of $\alpha$. The relative change in this product with variation of $\alpha$ is $O\left(H_{m}^{2}\right) \ll 1$ and is comparable to its change under variation of factor ordering. The latter represents a genuine uncertainty of the stochastic approach.

[45] Goncharov, Linde, and Mukhanov, Int. J. Mod. Phys. A 2, 561 (1987).

[46] Y. Nambu and M. Sasaki, Phys. Lett. B 219, 240 (1989).

[47] M. Mijic, Phys. Rev. D 42, 2469 (1990).

[48] S. Winitzki (unpublished).

[49] P. Ginsparg and M. Perry, Nucl. Phys. B222, 245 (1983); R. Bousso and S. W. Hawking, Phys. Rev. D 54, 6312 (1996).

[50] D. A. Samuel and W. A. Hiscock, Phys. Rev. D 44, 3052 (1991).

[51] Linde et al. $[19,18]$ compared the number of civilizations in different types of thermalized regions at a given moment of time $t$, rather than at all times prior to $t$. The results of this approach are also strongly gauge-dependent.

[52] One could choose a comoving volume which is initially is in the false vacuum and use the $\epsilon$-prescription to calculate the probabilities, disregarding the recycling. However, such a procedure appears somewhat artificial in the case of a recycling universe.

[53] D. S. Salopek and J. R. Bond, Phys. Rev. D 43, 1005 (1991).

[54] A similar suggestion has been made independently by Max Tegmark (private communication). 